\title{
On the structure of some groups having finite contranormal subgroups*
}

\author{
L. A. Kurdachenko and N. N. Semko
}

Dedicated to the 60th anniversary of the Department of Algebra and Mathematical Logic of Taras Shevchenko National University of Kyiv

A BStRACT. Following J.S. Rose, a subgroup $H$ of the group $G$ is said to be contranormal in $G$, if $G=H^{G}$. In a certain sense, contranormal subgroups are antipodes to subnormal subgroups. We study the structure of Abelian-by-nilpotent groups having a finite proper contranormal $p$-subgroup.

\section{Introduction}

Let $G$ be a group, and let $H$ be a subgroup of $G$. Denote, by $H^{G}$, the normal closure of $H$ in the group $G$, i.e., the least normal subgroup of $G$, including $H$. We have the inclusions $H \leqslant H^{G} \leqslant G$. The following polar situation appears here: $H=H^{G}$ and $H^{G}=G$. In a first case, a subgroup $H$ is normal in $G$. A subgroup $H$ of the group $G$ is called contranormal in $G$, if $G=H^{G}$.

The term "a contranormal subgroup" was introduced by J.S. Rose in paper [9].

As we can see by the definition, contranormal subgroups are antipode to normal subgroups: a contranormal subgroup $H$ of a group $G$ is normal if and only if $H=G$.

${ }^{*}$ The first author is supported by the National Research Foundation of Ukraine (grant no. 2020.02/0066).

2020 MSC: 20E99, 20F18, 20F19.

Key words and phrases: contranormal subgroups, Abelian-by-nilpotent groups, hypercenter of a group, $G$-eccentric subgroups, rationally irreducible subgroups. 
Starting from the normal closure, we obtain the following natural descending series:

$$
G=H_{0} \geqslant H_{1} \geqslant H_{2} \geqslant \ldots H_{\alpha} \geqslant H_{\alpha+1} \geqslant \ldots H_{\gamma},
$$

where $H_{1}=H^{G}, H_{2}=H^{H_{1}}, H_{\alpha+1}=H^{H_{\alpha}}$ for all ordinals $\alpha<\gamma$ and $H_{\lambda}=\bigcap_{\mu<\lambda} H_{\mu}$ for limit ordinals $\lambda, H_{\gamma}=H^{H_{\gamma}}$.

This series is called the lower normal closure series, and its last term $H_{\gamma}$ is called the lower normal closure of the subgroup $H$. We note that $H$ is contranormal in its lower normal closure.

The subgroup $H$ is called descendent in $G$, if $H$ coincides with its lower normal closure. The subgroup $H$ is called subnormal in $G$, if $H$ is descendent, and its lower normal closure series is finite. As we can see from this definition, the contranormal subgroups are antipodes to the descendent and subnormal subgroups.

In particular, the groups whose subgroups are subnormal do not include a proper contranormal subgroup.

In the present work, we will consider how the presence of small contranormal subgroups in a group affects its structure.

We note that if $H$ is a contranormal subgroup of $G$, then every subgroup $K$ including $H$ is contranormal in $G$. In particular, if $H$ and $L$ are contranormal subgroups of $G$, then a subgroup $\langle H, L\rangle$ also is contranormal in $G$. However, the intersection of two contranormal subgroups not always is contranormal. For example, in the group $A_{4}$, every Sylow 3 -subgroup is contranormal, but the intersection of every two Sylow 3-subgroups of $A_{4}$ is trivial, so that it is not contranormal. Note also that if $M$ is a maximal subgroup of $G$, which is not normal, then, clearly, $M$ is a contranormal subgroup of $G$.

The study of the groups having finite contranormal subgroups was started in paper [6], where the locally nilpotent Abelian-by-finite groups having finite contranormal subgroups were considered.

In the present paper, we will describe an arbitrary Abelian-by-nilpotent group having a finite contranormal $p$-subgroup, where $p$ is a prime. Before the formulation of the basic result of the present paper, we give the necessary definitions.

Let $G$ be a group, and let $A$ and $D$ be the normal subgroups of $G$ such that $A \leqslant D$. The factor $D / A$ is called $G$-central, if $C_{G}(D / A)=G$. The factor $D / A$ is called $G$-eccentric, if $C_{G}(D / A) \neq G$. 
A normal subgroup $A$ of the group $G$ is called $G$-hypereccentric, if it has an ascending series of $G$-invariant subgroups

$$
\langle 0\rangle=E_{0} \leqslant E_{1} \leqslant \ldots E_{\alpha} \leqslant E_{\alpha+1} \leqslant \ldots E_{\gamma}=A,
$$

and each factor $E_{\alpha+1} / E_{\alpha}$ is $G$-eccentric and $G$-chief for every $\alpha<\gamma$.

Let $G$ be a group, and let $A$ be an Abelian torsion-free subgroup of $G$. Then $A$ is called $G$-rationally irreducible, if, for every non-trivial $G$-invariant subgroup $D$ of $A$, the factor $A / D$ is periodic.

The main result of this paper is the following proposition.

Theorem. Let $G$ be a group, and let $A$ be a normal Abelian subgroup of $G$ such that $G / A$ is nilpotent. Suppose that $G$ includes a finite contranormal p-subgroup $C$, where $p$ is a prime. Then $G$ satisfies the following conditions:

(i) $G=D \lambda P$, where $D$ is a normal Abelian subgroup, and $P$ is a p-subgroup;

(ii) $P$ is a hypercentral Abelian-by-finite p-subgroup having a finite contranormal subgroup;

(iii) $D=D^{p}$;

(iv) $\operatorname{Tor}(D)=Q$ is a $G$-hypereccentric $p^{\prime}$-subgroup;

(v) every $G$-chief $p^{\prime}$-factor of $D$ is $G$-eccentric;

(vi) if $K, L$ are pure $G$-invariant subgroup of $D$ such that $T \leqslant K \leqslant L$, then $L / K=[L / K, G]$;

(vii) $D$ has an ascending series of $G$-invariant subgroups

$$
T=D_{0} \leqslant D_{1} \leqslant \ldots D_{\alpha} \leqslant D_{\alpha+1} \leqslant \ldots D_{\gamma}=D
$$

each factor $D_{\alpha+1} / D_{\alpha}$ of which is torsion-free $G$-eccentric and $G$ rationally irreducible, for every $\alpha<\gamma$.

We note that the hypercentral Abelian-by-finite $p$-subgroup having a finite contranormal subgroup was described in paper [6].

\section{Preliminary results}

Lemma 1. Let $G$ be a group. Then

(i) If $C$ is a contranormal subgroup of $G$, and if $H$ is a normal subgroup of $G$, then $C H / H$ is a contranormal subgroup of $G / H$.

(ii) If $H$ is a normal subgroup of $G$, and if $C$ is a subgroup of $G$ such that $H \leqslant C$ and $C / H$ is a contranormal subgroup of $G / H$, then $C$ is a contranormal subgroup of $G$.

(iii) If $C$ is a contranormal subgroup of $G$, and if $D$ is a contranormal subgroup of $C$, then $D$ is a contranormal subgroup of $G$.

These assertions are obvious. 
It is convenient to use, in what follows, the language of the modules over group rings.

Let $G$ be a group, let $R$ be a ring, and let $A$ be an $R G$-module. If $B$ and $D$ be the $R G$-submodules of $A$ such that $B \leqslant D$, then the factor $D / B$ is called $G$-central, if $C_{G}(D / B)=G$, and $G$-eccentric, if $C_{G}(D / B) \neq G$.

Define the upper $G$-central series of $A$,

$$
\langle 0\rangle=C_{0} \leqslant C_{1} \leqslant \ldots C_{\alpha} \leqslant C_{\alpha+1} \leqslant \ldots C_{\gamma},
$$

where

$$
\begin{gathered}
C_{1}=\{a \mid a \in A \text { and } a g=a \text { for all elements } g \in G\}=\zeta_{G}(A), \\
C_{\alpha+1} / C_{\alpha}=\zeta_{G}\left(A / C_{\alpha}\right) \text { for all ordinals } \alpha<\gamma \\
C_{\lambda}=\bigcup_{\mu<\lambda} C_{\mu} \text { for limit ordinals } \lambda<\mu \\
\zeta_{G}\left(A / C_{\gamma}\right)=\langle 0\rangle .
\end{gathered}
$$

We note that every member of this series is an $R G$-submodule.

The last term $C_{\gamma}$ of this series is called the upper $G$-hypercenter of $A$ and is denoted by $\zeta_{G}^{u p p e r}(A)$.

A module $A$ is called $G$-hypercentral, if $A=\zeta_{G}^{u p p e r}(A)$. In this case, if $\gamma$ is finite, then we say that $A$ is $G$-nilpotent.

The $R G$-module $A$ is called $G$-hypereccentric, if it has an ascending series of $R G$-submodules

$$
\langle 0\rangle=E_{0} \leqslant E_{1} \leqslant \ldots E_{\alpha} \leqslant E_{\alpha+1} \leqslant \ldots E_{\gamma}=A,
$$

each factor $E_{\alpha+1} / E_{\alpha}$ of which is $G$-eccentric and $R G$-chief, for every $\alpha<\gamma$.

We say that the $R G$-module $A$ has the $Z(G)$-decomposition, if $A=C \oplus$ $E$, where $C$ is the upper $R G$-hypercenter of $A$, and $E$ is a $G$-hypereccentric $R G$-submodule of $A$. We remark that if this decomposition exists, then it is unique [8, Chapter 10].

Lemma 2. Let $G$ be a finite nilpotent group, $F$ be a field, and $A$ be an $F G$-module. Then $A$ has the $Z(G)$-decomposition.

Proof. Let $\mathfrak{S}$ be a family of all $F$-subspaces of $A$ having a finite dimension. Since $G$ is finite, the $F G$-submodule generated by every member of $\mathfrak{S}$ has a finite dimension over $F$. Thus, $A$ has a local family $\mathfrak{L}$ of $F G$-submodules having a finite dimension over $F$. In particular, every member $D$ of $\mathfrak{L}$ has a finite $F G$-composition series. Then $D$ has the $Z(G)$-decomposition [7, Corollary 2.5]. Since all members of $\mathfrak{L}$ generate an $F G$-module $A, A$ has the $Z(G)$-decomposition [8, Corollary 10.6]. 
Let $G$ be a group, and let $A$ be a $Z G$-module. Then $A$ is called $G$ rationally irreducible, if, for every non-zero $Z G$-submodule $D$ of $A$, the factor-module $A / D$ is $Z$-periodic.

Corollary 1. Let $G$ be a finite nilpotent group, and let $A$ be a $Z G$-module, whose additive group is torsion-free. Then $A$ includes the $Z G$-submodule $D$ satisfying the following conditions:

(i) the factor-module $A / D$ is hypercentral;

(ii) $D$ is a pure subgroup of $A$;

(iii) $D$ has an ascending series of pure $Z G$-submodules

$$
\langle 0\rangle=D_{0} \leqslant D_{1} \leqslant \ldots D_{\alpha} \leqslant D_{\alpha+1} \leqslant \ldots D_{\gamma}=D,
$$

each factor $D_{\alpha+1} / D_{\alpha}$ of which is $G$-eccentric and $G$-rationally irreducible, for every $\alpha<\gamma$;

(iv) if $K, L$ are pure $Z G$-submodule of $D$ such that $K \leqslant L$, and if the factor $L / K$ is $G$-rationally irreducible, then $L / K$ is $G$-eccentric.

Proof. Let $B$ be a divisible hull of $G$. We can transform $B$ in a natural way in the $Q G$-module. By Lemma 2, the $Q G$-module $B$ has the $Z(G)$ decomposition: $B=C \oplus E$, where $C$ is the upper $G$-hypercenter of $B$, and $E$ is the maximal $G$-hypereccentric $Q G$-submodule. Then $E$ has an ascending series of $Q G$-submodules

$$
\langle 0\rangle=E_{0} \leqslant E_{1} \leqslant \ldots E_{\alpha} \leqslant E_{\alpha+1} \leqslant \ldots E_{\gamma}=A,
$$

each factor $E_{\alpha+1} / E_{\alpha}$ of which is $G$-eccentric and $Q G$-chief, for every $\alpha<\gamma$.

Put $D=A \cap E, D_{\alpha}=A \cap E_{\alpha}, \alpha<\gamma$. Then a $G$-isomorphism $A / D=A /(A \cap E) \cong(A+E) / E \leqslant B / E$ shows that $A / D$ is $G$-hypercentral. Moreover, the additive group of $A / D$ is torsion-free. We note also that every $D_{\alpha}$ is a pure subgroup of $A, \alpha<\gamma$. Let $U$ be a $Z G$-submodule of $D_{\alpha+1}$ such that $D_{\alpha} \leqslant U$ and $D_{\alpha} \neq U$. Let $V$ be a divisible hull of $U$, then $V / U$ is periodic. Moreover, $V$ is a $Q G$-submodule, and the inclusion $U \leqslant D_{\alpha+1}$ implies that the divisible hull of $E_{\alpha+1}$ of $D_{\alpha+1}$ includes $V$. The facts that $D_{\alpha} \leqslant U$ and $D_{\alpha} \neq U$ yield $E_{\alpha} \leqslant V$ and $E_{\alpha} \neq V$. Since $E_{\alpha+1} / E_{\alpha}$ is a $Q G$-chief factor, $V=E_{\alpha+1}$. Since the additive group $V / U$ is periodic, $D_{\alpha+1} / U$ is also periodic. This means that $D_{\alpha+1} / D_{\alpha}$ is a $G$-rationally irreducible factor.

We note that $C_{G}\left(D_{\alpha+1} / D_{\alpha}\right)=C_{G}\left(E_{\alpha+1} / E_{\alpha}\right)$, so that each factor $D_{\alpha+1} / D_{\alpha}$ is $G$-eccentric, $\alpha<\gamma$.

Finally, let $K$ and $L$ be the pure $Z G$-submodules of $D$ such that $K \leqslant L$, and let the factor $L / K$ be $G$-rationally irreducible. Suppose that $L / K$ is $G$-central. Choose an element $y \in L$ such that $y \notin K$. Denote, 
by $Y$, the $Z G$-submodule of $D$ generated by an element $y$. Since $G$ is finite, the additive group of $Y$ is finitely generated. Let $X$ be the divisible hull of $Y$. An inclusion $Y \leqslant D$ implies that $X \leqslant E$. We can consider $X$ as a $Q G$-submodule of $E$. Since $Y /(Y \cap K)$ is torsion-free, the divisible hull $W$ of $Y \cap K$ does not coincide with $X$. Clearly, $C_{G}(Y /(Y \cap K))=C_{G}(X / W)$. It follows that a $Q G$-submodule $X$ has a central $Q G$-factor. Being finitely generated, the $Q G$-module $X$ over a finite group $G$ has a finite $Q G$ composition series. Then $X$ has the $Z(G)$-decomposition [7, Corollary 2.5]. The fact that $X$ has a non-trivial $G$-central factor implies that $\zeta_{G}(X) \neq\langle 0\rangle$, and we obtain a contradiction with the choice of $E$. This contradiction proves (iv).

Lemma 3. Let $G$ be a finite nilpotent group, and let $A$ be a $Z G$-module. If the additive group $A$ is periodic, then $A$ has the $Z(G)$-decomposition.

Proof. If $B$ is a finite subgroup of $A$, then the finiteness of $G$ implies that a $Z G$-submodule generated by $B$ is finite. It follows that $A$ has a local family $\mathfrak{S}$ consisting of finite $Z G$-submodules. In particular, every member $D$ of $\mathfrak{S}$ has finite $F G$-composition series. Then $D$ has the $Z(G)$ decomposition [7, Corollary 2.5]. Since all members of $\mathfrak{S}$ generate a $Z G$-module $A, A$ has the $Z(G)$-decomposition [8, Corollary 10.6].

Lemma 4. Let $G$ be a group, and let $K$ be a normal subgroup of $G$ such that $G / K$ is nilpotent. If $S$ is a contranormal subgroup of $G$, then $G=K S$.

Proof. We note that, in the nilpotent group, every subgroup is subnormal. It follows that a proper subgroup of the nilpotent group cannot be contranormal. By Lemma $1, S K / K$ is a contranormal subgroup of the nilpotent group $G / K$. By the above remark, $S K / K=G / K$ or $S K=G$.

Corollary 2. Let $G$ be a group, and let $A$ be a normal Abelian torsionfree subgroup of $G$ such that $G / A$ is nilpotent. If $G$ includes a finite contranormal subgroup $C$, then $G=A \lambda C$, and every $G$-irreducible torsion-free factor of $A$ is $G$-eccentric.

Proof. By Lemma 4, $G=A C$. Since $A$ is torsion-free, $A \cap C=\langle 1\rangle$. By Corollary 1, $A$ includes a pure $G$-invariant subgroup $D$ such that the upper hypercenter of $G / D$ includes $A / D$, and if $K$ and $L$ are pure $G$-invariant subgroup of $D$ such that $K \leqslant L$, and if the factor $L / K$ is $G$-rationally irreducible, then $L / K$ is $G$-eccentric.

Suppose that $D \neq A$. Since $G / A$ is nilpotent, $G / D$ is hypercentral. In the hypercentral group $G / D$, the set $T / D$ of all elements having finite orders is a characteristic subgroup of $G / D$ (see, e.g., [4, Corollary 1.2.6 
and Proposition 1.2.11]). The fact that $G / D$ is not periodic implies that $G / D \neq T / D$. But, in this case, $C D / D \leqslant T / D$ and $C^{G} \leqslant T$, because $T$ is normal in $G$. In particular, $C^{G} \neq G$, and we obtain a contradiction. This contradiction proves an equality $A=D$.

Let $U$ and $V$ be the $G$-invariant subgroups of $A$ such that $U \leqslant V$, and $V / U$ is torsion-free and $G$-irreducible. Suppose that $C_{G}(V / U)=$ $G$. Denote, by $R / U$, the periodic part of $A / U$. Since $U$ is $G$-invariant, $R$ is also $G$-invariant. The fact that $V / U$ is torsion-free implies that $\langle 1\rangle=V / U \cap R / U$. We have $V R / R \cong V /(V \cap R)=V / U$, in particular, $C_{G}(V R / R)=G$. Let $Q / R$ be a pure envelope of $V R / R$. Clearly, a subgroup $Q$ is $G$-invariant and $C_{G}(V R / R)=C_{G}(Q / R)$. In particular, $C_{G}(Q / R)=G$, and we obtain a contradiction. This contradiction proves that the factor $V / U$ is $G$-eccentric.

Lemma 5. Let $G$ be a group, and let $A$ be a normal Abelian torsion-free subgroup of $G$ such that $G / A$ is nilpotent. Suppose that $G$ includes a finite contranormal $p$-subgroup $C$, where $p$ is a prime. If $C \cap A=\langle 1\rangle$, then $A=A^{p}$.

Proof. Suppose the contrary. Let $D=A^{p} \neq A$. Then $C D / D$ is a proper subgroup of $G / D$. Being bounded, the Abelian-by-finite $p$-group, $G / D$ is nilpotent (see, e.g., [1]). By Lemma $1, C D / D$ is a contranormal subgroup of $G / D$. But a nilpotent group does not include proper contranormal subgroups, and we obtain a contradiction. This contradiction proves the equality $A^{p}=A$.

Lemma 6. Let $G$ be a group, and let $A$ be a normal Abelian torsionfree subgroup of $G$ such that $G / A$ is nilpotent. If $G$ includes a finite contranormal p-subgroup $C$, where $p$ is a prime, then every $G$-chief $p^{\prime}$ factor of $A$ is $G$-eccentric.

Proof. By Corollary 2, $G=A \lambda C$, in particular, $G / A$ is finite. Let $U$ and $V$ be the $G$-invariant subgroups of $A$ such that $U \leqslant V$ and a factor $V / U$ is $G$-chief. The finiteness of $G / A$ implies that $V / U$ is finite. The fact that $V / U$ is $G$-chief implies that $V / U$ is a $q$-group for some prime $q \neq p$.

Suppose the contrary. Let $G=C_{G}(V / U)$. Denote, by $T / U$, the periodic part of $G / U$ and, by $Q / U$, the Sylow $q$-subgroup of $A / U$. Since $U$ is $G$ invariant, $T$ and $Q$ are also $G$-invariant. The inclusion $V / U \leqslant \zeta_{G}(A / U)$ implies that the upper $G$-hypercenter of $Q / U$ is not trivial. We have $T / U=$ $Q / U \times R / U$, where $R / U$ is the Sylow $q^{\prime}$-subgroup of $A / U$. We note that a subgroup $R$ is also $G$-invariant. The isomorphism $T / R \cong Q / U$ implies that the upper $G$-hypercenter $Z / R$ of $T / R$ is not trivial. Lemma 3 implies 
that a factor $T / R$ has the $Z(G)$-decomposition: $T / R=Z / R \times E / R$. We note that a subgroup $E$ is $G$-invariant. Thus the factor $T / E$ is not trivial, periodic, and $G$-hypercentral. Since $q \neq p$, it follows that $T / E \leqslant \zeta_{G}(A / E)$.

Suppose that $T=A$. Then the inclusion $T / E \leqslant \zeta_{G}(A / E)$ implies that $G / E$ is nilpotent. Since $A \cap C=\langle 1\rangle, C E / E$ is a proper subgroup of $G / E$. On the other hand, Lemma 1 shows that $C E / E$ is a contranormal subgroup of $G / E$, and we obtain a contradiction with the fact that the nilpotent group does not include a proper contranormal subgroup. This contradiction proves that $T \neq A$.

The choice of $T$ shows that a factor $A / T$ is torsion-free. Choose, in $A / E$, a $G$-invariant subgroup $S / E$ which is maximal by the property $T / E \cap S / E=\langle 1\rangle$. Suppose that the factor $A / S$ contains an element $b S$ having the infinite order. Put $B / S=\langle b S\rangle^{G / S}$. By its choice, $B / S$ is infinite. Since $G / A$ is finite, $B / S$ is finitely generated. Then $B / S=$ $W / S \times D / S$, where $W / S$ is a finite periodic part of $B / S$. Let $|W / S|=t$. Then $Y / S=(B / S)^{t}=(D / E)^{t}$ is a $G$-invariant subgroup. The inclusion $Y / S \leqslant D / S$ shows that $Y / S$ is torsion-free. Then $Y / S \cap T S / S=\langle 1\rangle$ so that $Y / E \cap T / E=\langle 1\rangle$, and we obtain a contradiction with a choice of $S$. This contradiction proves that $A / S$ is periodic. It follows that $A / S$ is a q-group.

The isomorphism $T / E \cong T S / S$ implies that the upper $G$-hypercenter $Z_{1} / S$ of $A / S$ is not trivial. Lemma 3 implies that the factor $A / S$ has the $Z(G)$-decomposition:

$$
A / S=Z_{1} / S \times E_{1} / S .
$$

We note that a subgroup $E_{1}$ is $G$-invariant. Thus, the factor $A / E_{1}$ is not trivial, periodic, and $G$-hypercentral. Using the above arguments, we again obtain a contradiction. This final contradiction shows that a factor $V / U$ is $G$-eccentric.

Lemma 7. Let $G$ be a group, and let $A$ be a normal Abelian torsion-free subgroup of $G$ such that $G / A$ is nilpotent. Suppose that $G$ includes a finite contranormal p-subgroup, where $p$ is a prime. If $D$ is a pure $G$-invariant subgroup of $A$, then $[D, G]=D$.

Proof. Suppose the contrary. Let $C=[D, G] \neq D$. First, we assume that the factor $D / C$ is not periodic. Denote, by $T / C$, the periodic part of $D / C$. Since $C$ is $G$-invariant, $T$ is also $G$-invariant. Choose, in the subgroup $D$, an element $b$ such that $b \notin T$. Then $\langle b T\rangle=\langle b T\rangle^{G / T}$. Denote, by $B_{1} /\langle b T\rangle$, the periodic part of $D /\langle b T\rangle$. Then $B_{1}$ is a pure subgroup of $D$. Hence, $D$ is pure in $A, B_{1}$ is a pure subgroup of $A$. Clearly, $C_{G}\left(B_{1} / T\right)=C_{G}(\langle b T\rangle)$, in particular, a factor $B_{1} / T$. The equality $r_{0}\left(B_{1} / T\right)=r_{0}(\langle b T\rangle)=1$ shows 
that $B_{1} / T$ is $G$-rationally irreducible, so that we obtain a contradiction with Corollary 1. This contradiction proves that $D / C$ must be periodic.

Lemma 6 shows that $D / C$ is a $p$-group. Since $D$ is a pure subgroup of $A$, Lemma 5 yields $D^{p}=D \cap A^{p}=D \cap A=D$. Hence, $D / C$ is a divisible $p$-group. Then $D / C$ is a direct product of Prüfer $p$-subgroups (see, e.g., [5, Theorem 23.1]). Let $P / C$ be some Prüfer $p$-subgroup of $D / C$. Then $A / C=P / C \times K / C$ for some subgroup $K / C$ (see, e.g., [5, Theorem 21.2]). Since $G / A$ is finite, $D / C$ includes a $G$-invariant subgroup $E / C$ such that $A / C=(P / C)(E / C)$, and the intersection $P / C \cap E / C$ has a finite exponent (see, e.g., [8, Corollary 5.11]). Then

$$
\begin{aligned}
A / E & \cong(A / C) /(E / C)=((P / C)(E / C)) /(E / C) \\
& \cong(P / C) /(P / C \cap E / C) \cong P / C .
\end{aligned}
$$

Thus, we obtain that $A / E$ is a Prüfer $p$-group, and $A / E$ is the $G$-central factor of $A$. Then $G / E$ is an infinite nilpotent group. On the other hand, Lemma 1 shows that $C E / E$ is a finite contranormal subgroup of $G / E$, and we obtain a contradiction with the fact that a nilpotent group does not include a proper contranormal subgroup. This contradiction shows that $[D, G]=D$.

Corollary 3. Let $G$ be a group, and let $A$ be a normal Abelian torsion-free subgroup of $G$ such that $G / A$ is nilpotent. Suppose that $G$ includes a finite contranormal $p$-subgroup $C$, where $p$ is a prime. If $K$ and $L$ are pure $G$-invariant subgroups of $A$ such that $K \leqslant L$, then $L / K=[L / K, G]$.

Lemma 8. Let $G$ be a group, and let $A$ be a normal Abelian subgroup of $G$ such that $G / A$ is nilpotent. Suppose that $G$ includes a finite contranormal p-subgroup $C$, where $p$ is a prime. If $\operatorname{Tor}(A)=T$ is a $p^{\prime}$-subgroup, then $T$ is G-hypereccentric.

Proof. By Lemma $4, G=A C$. It follows that $G / A$ is a $p$-group. By Lemma 3, a subgroup $T$ has the $Z(G)$-decomposition: $T=Z \times E$, where $Z$ is the upper $G$-hypercenter of $T$, and $E$ is a maximal $G$-invariant $G$-hypereccentric subgroup of $T$.

Suppose the contrary. Let $Z \neq\langle 1\rangle$. Further, we will consider a factorgroup $G / E$. Therefore, without loss of generality, we may assume that $E=\langle 1\rangle$. In other words, we will suppose that the upper $G$-hypercenter of $G$ includes $T$.

Since $T$ is a $p^{\prime}$-subgroup, $T \leqslant \zeta_{G}(A)$. Choose, in $A$, the $G$-invariant subgroup $S$ which is maximal by the property $T \cap S=\langle 1\rangle$. As in a proof of Lemma 6, we obtain that $A / S$ is a periodic group, and $\Pi(A / S)=\Pi(T)$. 
Using Lemma 3, we obtain that $A / S$ has the $Z(G)$-decomposition: $A / S=$ $Z_{1} / S \times E_{1} / S$, where $Z_{1} / S$ is the upper $G$-hypercenter of $A / S$, and $E_{1} / S$ is a maximal $G$-invariant $G$-hypereccentric subgroup of $A / S$. The obvious inclusion $T S / S \leqslant Z_{1} / S$ and the choice of $S$ imply that $A / S=Z_{1} / S$. Since $A / S$ is a $p^{\prime}$-subgroup, $A / S=\zeta_{G}(A / S), G / S$ is a nilpotent group. Moreover, the fact that $A / S$ is a $p^{\prime}$-subgroup implies that $C S / S$ is a proper subgroup of $G / S$. On the other hand, Lemma 1 shows that $C S / S$ is a contranormal subgroup of $G / S$, and we obtain a contradiction with the fact that the nilpotent group does not include a proper contranormal subgroup. This contradiction shows that $T$ is $G$-hypereccentric.

Lemma 9. Let $G$ be a group, and let $A$ be a normal Abelian subgroup of $G$ such that $G / A$ is nilpotent. Suppose that $G$ includes a finite contranormal p-subgroup $C$, where $p$ is a prime. If $\operatorname{Tor}(A)=T$ is a p-subgroup, then $G=D \lambda P$, where $D$ is a normal torsion-free Abelian subgroup, and $P$ is a p-subgroup.

Proof. By Lemma 4, $G=A C$. We note that $C$ is a $p$-subgroup, $C \cap$ $A=C \cap T$. Therefore, without loss of generality, we can suppose that $C \cap A=\langle 1\rangle$.

By Lemma $5, A=A^{p}$. Since the periodic part $T$ of $A$ is pure, $T^{p}=T$. The fact that $T$ is a $p$-subgroup implies that $T$ is divisible. Then $A=T \times U$ for some subgroup $U$ (see, e.g., [5, Theorem 21.2]). Since $G / A$ is finite, $A$ includes a $G$-invariant subgroup $V$ such that $A=T V$, and the intersection $T \cap V$ has a finite exponent $r=p^{t}$, where $p^{t}=|G / A|$ (see, e.g., [8, Corollary 5.11]). We have $V=(T \cap V) \times D$ for some subgroup $D$ (see, e.g., [5, Theorem 27.5]). It follows that $V^{t}=D^{t}$, in particular, a subgroup $D^{t}$ is $G$-invariant. The isomorphism

$$
D \cong V /(T \cap V) \cong V T / T=A / T
$$

and Lemma 5 imply that $D=D^{p}$. Then $D=D^{t}$. Thus, a subgroup $D$ is $G$-invariant. The choice of $D$ shows that $D$ is torsion-free. Furthermore,

$$
A=T V=T((T \cap V) \times D)=T \times D .
$$

Therefore, $G=A C=(D T) C=D \lambda(T C)$, where $P=T C$ is a $p$-subgroup of $G$.

\section{Proof of the main result}

Let $T=\operatorname{Tor}(A)$. We have $T=R \times Q$, where $R$ is the Sylow $p$-subgroup of $T, Q$ is the Sylow $p^{\prime}$-subgroup of $T$. The isomorphism $Q \cong T / R$ and 
Lemma 8 show that $Q$ is $G$-hypereccentric. Using Lemma 9, we obtain the decomposition $G / Q=D / Q \lambda S / Q$, where $S / Q$ is a $p$-subgroup, $D / Q$ is an Abelian normal torsion free group. In particular, $\operatorname{Tor}(D)=Q$. Since $Q$ is a normal Sylow $p^{\prime}$-subgroup of $S, S=Q \lambda P$ where $P$ is a Sylow $p$-subgroup of $S$ (see, e.g., [3, Theorem 2.4.5]). Being Abelian-by-finite $p$-subgroup, $P$ is hypercentral (see, e.g., [2, Proposition I.1.7]). Hence, $G=D S=D(Q P)=D \lambda P$. The isomorphism $P \cong G / D$ shows that the subgroup $P$ has a finite contranormal subgroup. The isomorphism $D / Q \cong A / T$ and Lemma 6 prove that every $G$-chief $p^{\prime}$-factor of $D / Q$ is $G$-eccentric. The fact that $Q$ is $G$-hypereccentric implies that every $G$-chief factor of $Q$ is $G$-eccentric. Corollary 3 proves (vi), and Corollary 1 yields (vii).

\section{References}

[1] G. Baumslag, Wreath products and p-groups, Math. Proc. Cambridge Philos. Soc., 55(3), 1959, 224-231.

[2] S.N. Chernikov, The Groups With Prescribed Properties of Systems of Subgroups, Nauka, Moscow, 1980.

[3] M.R. Dixon, Sylow Theory, Formations and Fitting Classes in Locally Finite Groups, World Scientific, Singapore, 1994.

[4] M.R. Dixon, L.A. Kurdachenko, I.Ya. Subbotin, Ranks of Groups. The Tools, Characteristics and Restrictions, Wiley, New York, 2017.

[5] L. Fuchs, Infinite Abelian Groups, I, Academic Press, New York, 1970.

[6] L.A. Kurdachenko, P. Longobardi, M. Maj, On the structure of some locally nilpotent groups without contranormal subgroups, 2020, arXiv:2006.02345v1 [math.GR].

[7] L.A. Kurdachenko, J. Otal, The rank of the factor-group modulo the hypercenter and the rank of the some hypocenter of a group, Centr. Eur. J. Math., 11(10), 2013, 1732-1741.

[8] L.A. Kurdachenko, J. Otal, I.Ya. Subbotin, Artinian modules over group rings, Frontiers in Mathematics, Birkhäuser, Basel, 2007.

[9] J.S. Rose, Nilpotent subgroups of finite soluble groups, Math. Z., 106(2), 1968, 97-112.

\section{CONTACT INFORMATION}

L. A. Kurdachenko Oles Honchar Dnipro National University, Gagarin avenue 72, 49010, Dnipro, Ukraine E-Mail(s): lkurdachenko@i.ua

N. N. Semko

Department of Mathematics, University of State Fiscal Service of Ukraine, Universitetska 31, 08205, Irpin, Kyiv region, Ukraine E-Mail(s): dr.mykola.semko@gmail.com

Received by the editors: 12.11 .2020 . 